\title{
REGISTRATION OF CT IMAGE AND FACIAL SURFACE DATA USING ADAPTIVE GENETIC ALGORITHM
}

\author{
Chung-Hsien Huang, Jiann-Der Lee, Jau-Hua Huang \\ Department of Electrical Engineering, Chang Gung University \\ Medical Augmented Reality Research Center, Chang Gung Memorial Hospital, \\ Tao-Yuan, Taiwan
}

\begin{abstract}
In this paper, we propose a novel registration method based on Adaptive Genetic Algorithm (adaptive-GA) to accomplish the registration task of computer tomography (CT) and its corresponding facial surface data. First, chain code method is utilized to represent the facial curve such that facial surface data obtained by a face scanner is efficiently reduced. Next, based on the concept of the genetic algorithm in continuous space (GACS), we improve its evolutional mechanisms of chromosomes' crossover and mutation to speed up the convergent process of general GA. From the experimental results, it is proved that the proposed registration method can be used for non-fiducials stereotactic brain surgeries and help surgeons to diagnose and treat brain disease correctly and conveniently.
\end{abstract}

Biomed Eng Appl Basis Comm, 2005(August); 17: 201-206.

Keywords: image registration, surface data, CT image, genetic algorithm, stereotactic surgery

\section{INTRODUCTION}

For the current brain surgery, surgeons often utilize computer tomography (CT), magnitude resonance imaging (MRI), or other medical imaging to find out where the anatomical target is and to evaluate the results of the surgical process. Generally, the surgical process can be described as follow. First, surgeons plan the cut size and depth after image scanning. Secondly, they settle the patient on the operating table and start to dig an opening through the skull. Finally, surgical tools are guided into patient' $\mathrm{s}$ brain through this opening and surgeons can do some treatments for the patient' $\mathrm{s}$ disease.

Received: Jan. 15, 2005; Accepted: July 8, 2005

Correspondence: Jiann-Der Lee, Professor

Department of Electrical Engineering, Chang Gung University, Tao-Yuan, Taiwan

E-mail: jdlee@mail.cgu.edu.tw
How to provide a suitable localization method is an important issue for brain stereotactic surgery. The stereotactic surgery, which is a surgical method, combined medical imaging with outer equipment or other stuffs can increase accuracy of surgical operation. Stereotactic surgery can be divided into fiducials, i.e. based on external objects introduced into the image space, and non-fiducials, i.e. based on the image information generated by the patient [1-10]. Fiducials methods rely on artificial objects such as stereotactic frame and markers attached to the patients. Uulike fiducials methods, non-fiducials methods rely on patient-generated image content only. Registration can be based on a limited set of some specific landmarks, on the image features, or directly on the image gray values. In this approach, we utilize two data sets, including CT and facial surface data obtained by a face scanner, to achieve the image registration task required for a non-fiducials stereotactic surgery. 
That is, CT provides the inner information of patient' $\mathrm{s}$ brain, and facial surface data provides the outer coordinate localization of surgery's tools.

Using the technique of image fusion can provide a good auxiliary to help the physicians in localizing the same region of interest on various images taken by a patient. Different images have their particular coordinates referred to specific photographic machines. And the registration procedure (or called coregistration, matching) is using a transformed matrix to locate source information from various imaging modalities on the same spatial coordinate. The registration procedure must be accurate enough to limit the amount of imprecision introduced. A meaningful image fusion is built on an accurate registration. Therefore, in this approach, we propose an adaptiveGA to solve the registration between CT and facial surface data such that the accuracy and safety of nonfiduciclas methods can be largely increased.

The remainder of this paper is organized as follow. Section 2 presents the proposed method for registration of CT Image and facial surface data using adaptive genetic algorithm. In this section, the strategy of feature extraction, the definition of fitness function, and the evolutional mechanisms are described in details. The experimental results using this approach are presented in section 3. At last, the conclusion is included in final section.

\section{METHOD}

The flowchart of the proposed scheme is illustrated in Fig. 1. There are two sets of source data, one is CT scanned before surgery and the other is facial surface data scanned during surgery. The aim of the feature point extraction step is to reduce a 3-D volume data of CT to a surface data and to trim a large number of surface data points. This step can largely save the huge computing cost of the following adaptive-GA. Finally, we utilize the technology of 3-D display to show the fusion result.

\subsection{Feature points extraction}

First, image preprocessing included segmentation and boundary tracing are used to extract a point set of CT surface data. Next, the chain code method is used for feature analysis. Fig. 2 represents the chain code notation, which is to encode the sequence of the previous and current point as relative direction. Its code means to "go straight", "turn left", etc. The blue points in Fig. 3(a) are selected randomly, while these in Fig. 3(b) are selected after performing chain code algorithm.

\subsection{Adaptive-GA}

Registration of two free-form surfaces can be considered as a search problem that included six parameters, i.e., three geometric translations and three rotation angles. Genetic algorithms are good at optimizing function with many local optimal points and have no restriction on the form of the fitness functions. GA is a population-oriented, store a sampled replica of the profile of the function being optimized, and provides important clues about the global structure of the function. GA is not only parallel but also global. The "fitness" information calculated by fitness function from different members is relied on various genetic operators, especially through selection, crossover and mutation mechanisms.

\subsubsection{Chromosomes 'coding}

The geometric relation between two data sets includes six parameters, three translations $\left(s_{x}, s_{y}, s_{z}\right)$ and three rotations $\left(\theta_{x}, \theta_{y}, \theta_{z}\right)$. The transform function can be defined as Eq. (1).

$$
\left[\begin{array}{l}
x \\
y \\
z
\end{array}\right]=R\left[\begin{array}{l}
x^{\prime} \\
y^{\prime} \\
z^{\prime}
\end{array}\right]+T
$$

where $\left[\begin{array}{lll}x & y & z\end{array}\right]^{T}$ and $\left[\begin{array}{lll}x^{\prime} & y^{\prime} & z^{\prime}\end{array}\right]^{T}$ are the coordinates after and before the transformation, respectively. $\boldsymbol{R}$ is the rotation matrix and $\boldsymbol{T}$ is the translation matrix.

Here, we define these six geometric parameters as a chromosome, as shown in Fig. 4.

\subsubsection{Fitness function}

Assume that the given two data sets to be matched are $P=\left\{p_{1}, p_{2}, \ldots, p_{\mathrm{m}}\right\}$ and $Q=\left\{q_{1}, q_{2}, \ldots, q_{\mathrm{n}}\right\}$, where $\boldsymbol{m}$ is not necessarily equal to $\boldsymbol{n}$. The size of $\boldsymbol{m}$ and $\boldsymbol{n}$ depend on the representation accuracy required to approximate the surfaces to be matched. As the previous section mentioned, if the registration parameters, i.e., rotation matrix $\boldsymbol{R}$ and translation matrix $\boldsymbol{T}$ are given, then for any point $\boldsymbol{p}_{\boldsymbol{i}}$ in $\boldsymbol{P}$, we can use the following criterion to determine its possible correspondent $\boldsymbol{q}_{i}$ in $\boldsymbol{Q}$ :

$$
q_{i}=\underset{q \in Q}{\arg \min }\left\|q-\left(R p_{i}+T\right)\right\|
$$

That is, Eq. (2) minimizes the Euclidean distance between the transformed point $R p_{i}+T$ and $\boldsymbol{q}$ in the $\boldsymbol{Q}$. A suitable transform means the distance error between $\boldsymbol{P}$ and $\boldsymbol{Q}$ is minimized. Therefore, the fitness function of GA to be minimized is described as Eq. (3).

$$
F=\sum\left\|p_{i}-q_{i}\right\|
$$



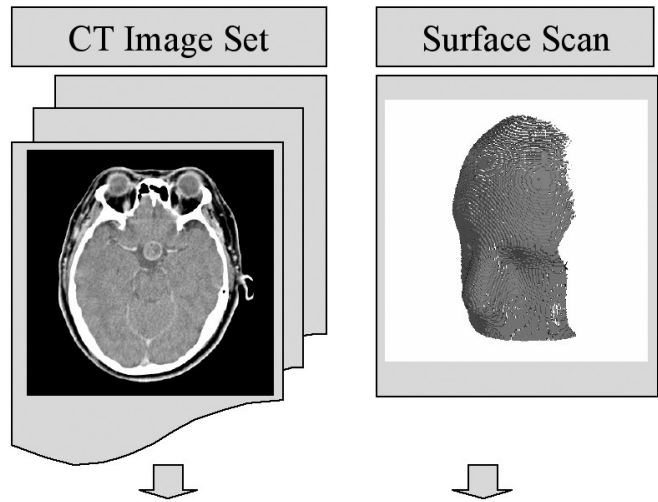

$\square$

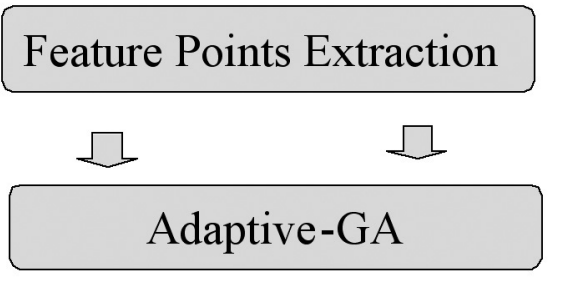

$\square$

\section{Fusion Result}

Fig. 1 The flowchart of data fusion

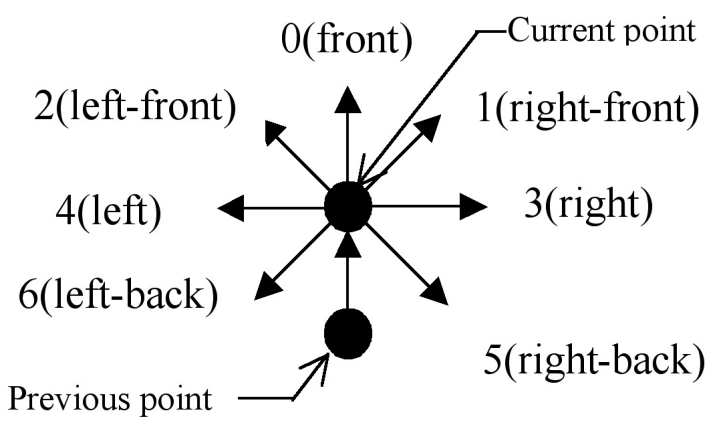

Fig. 2 Relative coordinates for a chain code scheme

\subsubsection{Evolutional mechanisms}

The spirit of GA is an evolutional process for getting the best next generation. It is noted that the quality of the evolutional mechanism of genes determined the performance of GA. The chromosome coding is usually by bit-string traditionally. But in the free-form surface registration, the solutions locate on a continuous space with six dimensions. Consequently, we use a floating point coding representation for GA, referred to as the Genetic Algorithm in Continuous Space (GACS) [11-12].

The GACS is described as below step by step, and

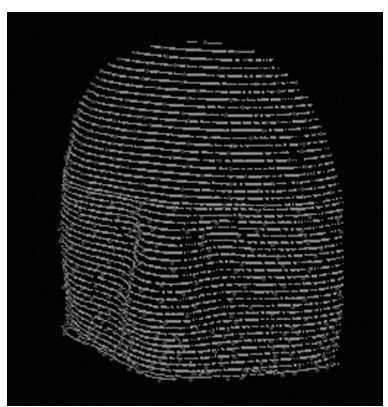

(a)

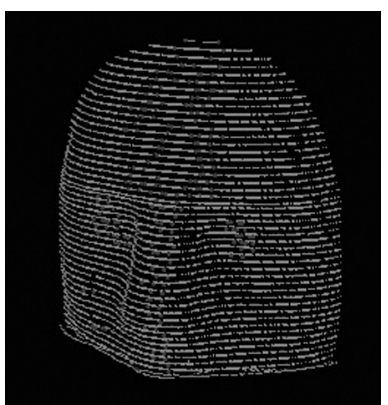

(b)
Fig. 3 The results after feature points extraction

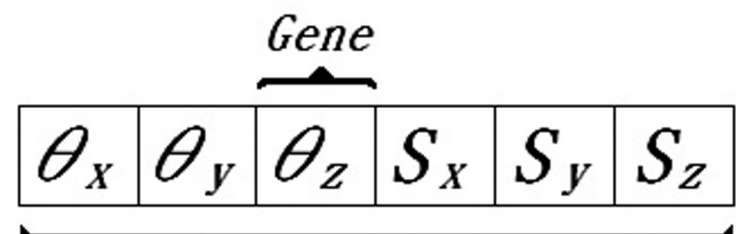

\section{Chromosome}

Fig. 4 The chromosomes ' coding

it is illustrated with Fig. 5 :

Step 1. Initial $\boldsymbol{N}$ chromosomes are generated randomly.

Step 2. Select the best chromosome by fitness function.

Step 3. There are three different ways mainly to generate the next generation, as show as in Fig. 6.

a. Selection: the best chromosome is preserved in the next generation.

b. Mutation: generate the $(N-1) / 2$ chromosomes of next generation by mutation of the best chromosome.

c. Crossover: generate the $(N-1) / 2$ chromosomes of next generation by crossover of the other chromosomes beside of the best chromosome.

Step 4. If the evolutional process trends to stable, the system will go to Step 5 and renew a generation randomly (re-initialization). Otherwise it will go back to Step 2.

Step 5. Since the first generation is given randomly, solutions usually fall into the local minimum easily. Then we use a mechanism called re-initialization to avoid this situation. The best chromosome is still prevented to next generation, and the other $(N-1)$ chromosomes are regenerated randomly. After some re-initialization, if the evolutional process trends to stable, the system will stop and get the final solution. Otherwise it will go back to Step 2. 


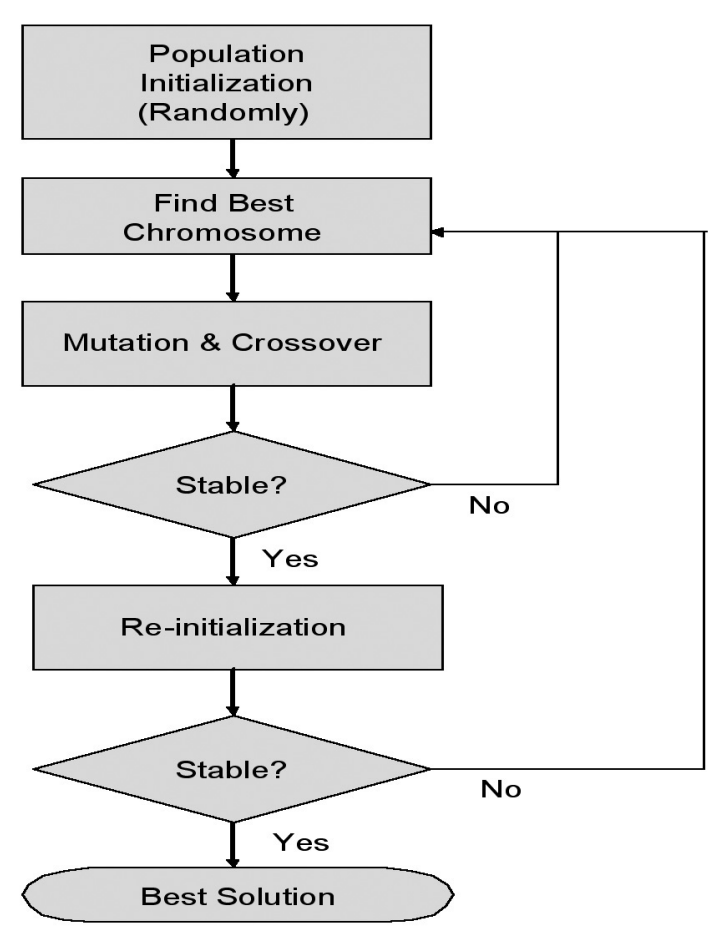

Fig. 5 The flowchart of adaptive-GA

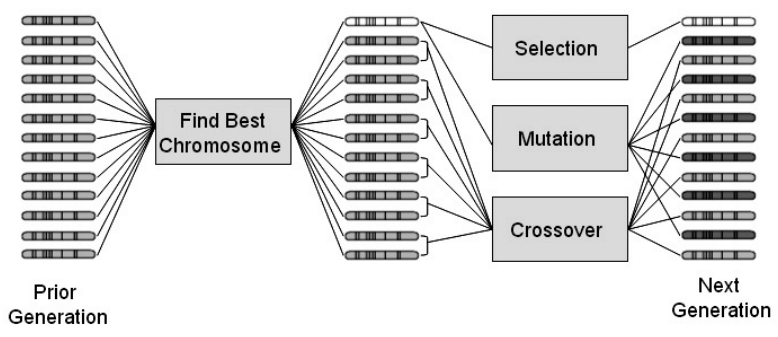

Fig. 6 Evolution mechanisms

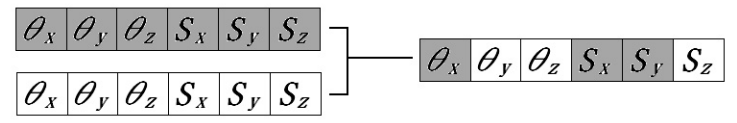

Fig. 7 Sketch of crossover

\subsubsection{Crossover}

Crossover is a mechanism, which swaps the genetic material between some selected couples of chromosomes. During the process of crossover, to start with, we select two chromosomes $\boldsymbol{A}$ and $\boldsymbol{B}$ to be parents, their fitness functions are $\boldsymbol{F}_{\boldsymbol{A}}$ and $\boldsymbol{F}_{\boldsymbol{B}}$, and $\boldsymbol{G}_{\boldsymbol{A}}$ and $\boldsymbol{G} \boldsymbol{B}$ are one of six genes of $\boldsymbol{A}$ and $\boldsymbol{B}$. Next, a new chromosome is generated along the order of six genes, and the probability of the selection of $\boldsymbol{G}_{\boldsymbol{A}}$ is
$p\left(G_{A}\right)=F_{B} /\left(F_{A}+F_{B}\right)$, the probability of the selection of $\boldsymbol{G}_{\boldsymbol{B}}$ is $p\left(G_{B}\right)=1-p\left(G_{A}\right)$. In other words, the gene of better parent has higher probability to pass to the children.

\subsubsection{Adaptive mutation}

Like the crossover, mutation is also an important mechanism to generate next population in GA. The mutation randomly modifies some genes of the chromosome and is responsible for the search space exploration. In this study, we only mutate the best chromosome and the effective mutation rate is $1 / 6$ since each chromosome has six genes. Normally, a gene always mutates between a suitable range or in its solution space, but we find that the range of mutation changed too large doesn't have great meanings and just generates useless chromosomes. Therefore, we designed an adaptive mutation to control the range of mutation.

The spirit of the adaptive mutation is that solutions are still farther from the best solution when generational results of fitness function converge rapidly. Therefore, the gene should mutate between a large range. On the other hand, if generational results of fitness function converge slowly, the gene should mutate between a small range to find a better chromosome. If the solution space of a gene $\boldsymbol{G}$ is $[-\boldsymbol{R}$, $\boldsymbol{R}$, the mutation range is $[\boldsymbol{G}-\boldsymbol{R} \boldsymbol{M}, \boldsymbol{G}+\boldsymbol{R} \boldsymbol{M}], \mathrm{F}$ is the result of fitness function of the initial generation, and $\boldsymbol{F}^{\prime}$ is the result of fitness function of the current generation. We can use Eq. (4) to express it.

$$
\left\{\begin{array}{l}
\text { if } \quad F \neq F^{\prime}, R_{M}=R\left(1-\exp \left(-k\left(\frac{1}{F-F^{\prime}}\right)\right)\right) \\
\text { else } \quad R_{M}=R
\end{array}\right.
$$

where $\mathrm{k}$ is a positive constant. It is noted that Eq. (4) is a monotonous decreasing function of $\left(F-F^{\prime}\right)$. The characteristic of this equation is demonstrated as Fig. 8.

\section{EXPERIMENTAL RESULTS}

As described in previous section, the aim of the proposed adaptive GA is to increase the convergent speed of traditional GA. Then, in the experiment, to evaluate the performance of the proposed Adaptive GA and general GA without adaptive mutation, we select one pair of CT image and its corresponding facial surface data for the registration. In this case, CT has 19210 points and reduces to nearly 300 points, while facial surface data has 195084 points and also reduces to nearly 300 points. From Fig. 9(a), we compare the 
total number of generations when the algorithm is converged. And Fig. 9(b) is the best fitness value of both algorithms. It is clear that adaptive GA converges quicker than GA, and the best fitness values of both algorithms are almost similar. Each algorithm has been executed 50 times repeatedly. More experimental results can be found in reference [13].

Besides, Fig. 10 reveals the total generation number required while using adaptive $\mathrm{GA}$ with the parameter $\mathrm{k}$ in Eq. (4) ranging from 10 to 200. As a result of Fig. 10, it is observed that the best result appears at $\boldsymbol{k}=30$, and it gets worse with the increase of $\boldsymbol{k}$. This information is helpful to select the appropriate $\mathrm{k}$ for adaptive GA.

\section{CONCLUSION}

In this paper, we proposed a registration method based on adaptive genetic algorithm to achieve the registration of CT and facial surface data. In general, the source data for registration always have more than hundred thousands of points. It may be a terrible costing for computer. We utilize chain code scheme to reduce a large number of surface data and it can reduce the executive time efficiently. With the concept of the genetic algorithm in continuous space (GACS), we improve its evolutional mechanisms of chromosomes' crossover and mutation to speed up the convergent process of GA. From the experimental results, it is proved that the proposed registration method can be used for non-fiducials stereotactic brain surgeries and help surgeons to diagnose and treat brain disease correctly and conveniently.

\section{ACKNOWLEDGEMENT}

This work was supported by Ministry of Economic Affairs, Taiwan. under Technology

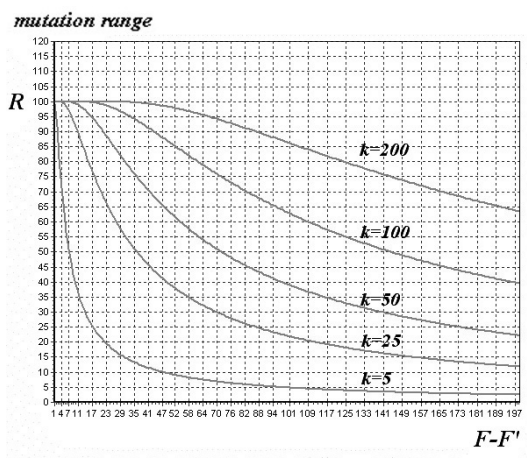

Fig. 8 The characteristic curve of Eq. (4)

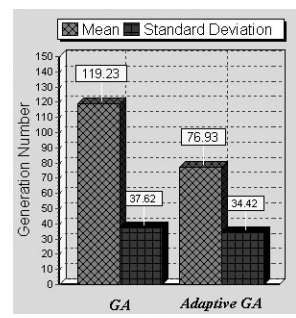

(a)

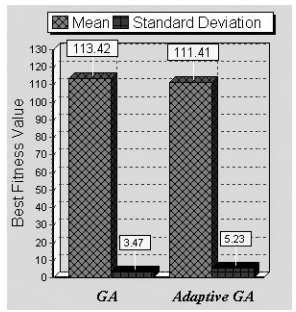

(b)
Fig. 9 Performance comparison of GA and adaptive GA

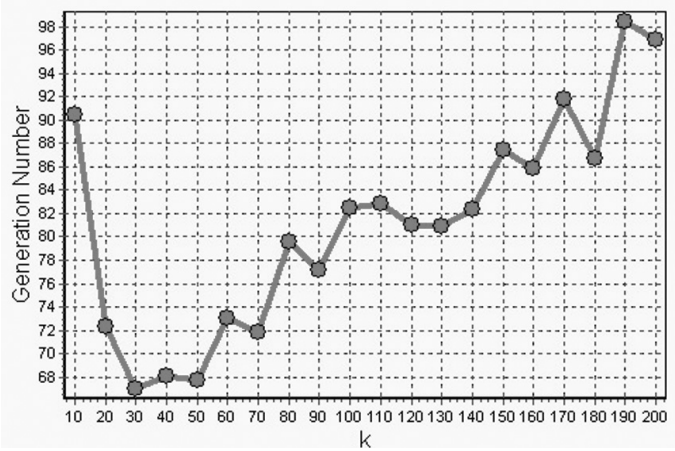

Fig. 10 The registration results using adaptive GA with different $k$

Development Program for Academia (TDPA) in the project of developing a Brain Medical Augmented Reality System with Grant No. 94-EC-17-A-19-S1035.

\section{REFERENCES}

1. A. Maintz, and A. Viergever, "A survey of medical image registration," Medical Image Analysis, 2:1-36, 1998.

2. S. Eberl, I. Kanno, R. R. Fulton, A. Ryan, B. F. Hutton and M. J. Fulham, "Automated interstudy image registration technique for SPECT and PET," J. Nucl. Med., 37:137-145, 1996.

3. C. Davatzikos, "Nonlinear registration of brain images using deformable models, " In Mathematical Methods in Biomedica Image Analysis, IEEE Computer Society Press, 94-103, 1996.

4. C. Davatzikos and J. L. Prince, "Brain image registration based on curve mapping," IEEE Workshop on Biomedical Image Analysis, IEEE Computer Society Press, 245-254, 1994.

5. R. Wasserman, J. C. Rajapakse and R. Acharya, Multimodality medical imaging for radiotherapy 
treatment planning," In IEEE Workshop on Biomedical Image Analysis, IEEE Computer Society Press, 235-244, 1994.

6. W. M. Wells III, P. Viola, H. Atsumi and R. Kikinis, "Multi-modalvolume registration by maximization of mutual information," In Medical Robotics and Computer Assisted Surgery, 55-62, Wiley, New York, 1995

7. W. M. Wells III, P. Viola, H. Atsumi, S. Nakajima and R. Kikinis, "Multi-modal volume registration by maximization of mutual information," Med. Image Anal., 1:35-51, 1997.

8. C. Davatzikos, J. L. Prince and R. N. Bryan, “ Image registration based on boundary mapping," IEEE Trans. Med. Imag., 15: 112-115, 1996.

9. G. Wang, N. D. Volkow, A. V. Levy, J. S. Fowler, J. Logan, D. Alexoff, R. J. Hitzemann, . and D. J. Schyler, "MR-PET image coregistration for quantitation of striatal dopamineDz receptors," J. Comput. Assis. Tomog, 20: 423-428, 1996.
10. S. Eberl, I. Kanno, R. R. Fulton, A. Ryan, B. F. Hutton and M. J. Fulham, "Automated interstudy image registration technique for SPECT and PET," J. Nucl. Med., 37: 137-145, 1996.

11. X. Qi, F. Palmieri, "Theoretical analysis of evolutionary algorithms with an infinite population size in continuous space, Part I: basic properties of selection and mutation. Part II: analysis of the diversification role of the crossover" , IEEE Trans. Neural Networks, 5: 102-129, 1994.

12. R. He, P. A. Narayana, "Global optimization of mutual information: application to threedimensional retrospective registration of magnetic resonance images" , Computerized Medical Imaging and Graphics, 26: 277-292, 2002. 